\title{
Do automated breast ultrasound and tomosynthesis have an effective role in dense breast evaluation?
}

Engy A. Ali ${ }^{i^{*}} \mathbb{D}$, Fatma Saeed ${ }^{1,2}$ and Lamiaa Adel ${ }^{1}$

\begin{abstract}
Background: Mammography plays a great role in reducing breast cancer mortality as it is the standard method of breast imaging and screening. But the accuracy of mammography performance reduces in cancer detection in women with dense breast due to the summation of images and overlapping of breast tissue. ABUS and tomosynthesis both recently help to detect breast cancer in dense breasted women. This prospective study was done in the female imaging unit and approved by its research and ethical committee; all the patients did an informed consent during the period from October 2018 to March 2019. The study was conducted on 38 patients with 38 lesions subjected to digital mammography, tomosynthesis and automated breast ultrasound (ABUS), who all had dense breast in mammography.

Results: Automated breast ultrasound (ABUS) showed 100\% in all sensitivity, specificity, negative predictive value (NPV), positive predictive value (PPV) as well as accuracy, while the digital mammography tomosynthesis showed 100\% in specificity, $87.5 \%$ in sensitivity, 100\% in PPV, $82.4 \%$ in NPV and $92.1 \%$ accuracy.
\end{abstract}

Conclusion: Automated breast ultrasound (ABUS) together with tomosynthesis makes a revolution in breast screening and detecting cancer in women with dense breasts.

Keywords: Automated breast 3D ultrasound, 3D tomosynthesis, Dense breast

\section{Background}

Breast cancer mortality rates have been reduced by using mammography as standard imaging method in screening. However, the summation images due to overlapping of breast tissue result in a reduction in the mammographic performance in screening and hiding the tumor shadow within the complicated dense glandular parenchyma, resulting in lowering the sensitivity of mammography [1]. Both automated breast ultrasound (ABUS) and tomosynthesis play a great role in early breast cancer detection as well as detecting different breast lesions, specially in

\footnotetext{
*Correspondence: drengyali78@gmail.com

1 Diagnostic and Intervention Radiology Department (Women's Imaging Unit), Kasr El-Ainy Hospital, Cairo University Hospitals, El-Manial,

Cairo 11956, Egypt

Full list of author information is available at the end of the article
}

dense breast parenchyma and that what had been evaluated and confirmed in this study.

\section{Aim of work}

The aim of this study is to evaluate the combination of tomosynthesis and ABUS to evaluate the diagnosis, detection and classification of breast lesions; it is potential to alleviate a limitation of mammography as masking of non-calcified cancer or distortion by other dense breast.

\section{Methods}

Patient population

In this prospective study, 38 consecutive women (ages 25-74, mean age $45+/-9$ SDyear) came for early screening or presented with palpable breast mass between October 2018 and March 2019 who showed either ACR 
C or ACR D, with 38 breast lesions (24 malignant and 14 benign).

\section{Data acquisition}

All of the females $(n=38)$ were subjected to both tomosynthesis and automated breast ultrasound. In tomosynthesis, both CC and MLO views were done using GE mammography. In ABUS, coronal and axial images were taken, and in the coronal planes, AP, medial and lateral views were done using GE Invenia ABUS system (GE Healthcare).

\section{Automated breast examination protocol design}

All patients did ABUS examination. All ABUS examinations were done using transducer of $15.3 \mathrm{~cm}$ and 6-15 MHz frequency. The transducer length is $15.3 \mathrm{~cm}$, with $6-15 \mathrm{MHz}$ frequency; the examination was done while the patient was in supine position placing a cushion beneath the shoulder to evenly spread the breast tissue with the nipple pointed toward the ceiling, putting hypoallergenic lotion on the breast mostly on the nipple.

To increase image quality and patient comfort, a disposal membrane was used to increase acoustic coupling and also by applying one of the three levels of compression. The women were told not to move or breath heavily during the scan which was continuous and automated.

Volume acquisitions were taken in the axial plane, from the inferior part of the breast with coronal and sagittal reconstruction. Three volumes were obtained for each breast anteroposterior volume with the nipple being in the center (putting a marker upon it), lateral volume involving the upper outer part of the breast with the nipple located at the intermedial corner as well as the medial volume involving the inner and inferior parts.

\section{Tomosynthesis}

Mammographic examination was performed using Senographe Essential, GE Healthcare, full-field digital mammography machine with 3D digital breast tomosynthesis. Taking both medio-lateral oblique and cranio-caudal views, images were assessed on the workstation.

\section{Image interpretation}

- Assessment of breast density and each lesion was evaluated including the size, site, mass or asymmetry and then classified into either benign or malignant using BIRADS lexicon classification. Assessment was done by two experienced radiologists, both unaware of the pathological data of each patient.

Pathological results were taken as the gold reference standard apart from 6 lesions that were proven by HHUS criteria to be benign ( 1 was hamartoma, 3 were diagnosed as fibroadenomas on follow-up and 2 simple cysts). Samples were taken by either fine needle aspiration cytology (FNAC), surgical excision and core biopsy.

\section{Statistical analysis}

Data were coded and entered using the SPSS (Statistical Package for the Social Sciences) version 24. Data were summarized using frequency (count) and relative frequency (percentage) for categorical data. Standard diagnostic indices including sensitivity, specificity, positive predictive value (PPV) and negative predictive value (NPV) were calculated. For comparing categorical data, chi-square $\left(\mathrm{x}^{2}\right)$ test was performed. Exact test was used instead when the expected frequency is less than 5. $P$ value less than 0.05 was considered as statistically significant.

\section{Results}

This prospective study involved 38 patients presented to $\mathrm{xxxx}$, their mean age was $(45 \pm 9) \mathrm{SD}$. Thirty-eight lesions were detected, 24 (63.2\%) cases were diagnosed as malignant, while $14(36.8 \%)$ were diagnosed as benign, using the pathological results as the gold standard reference except for 6 lesions that were proved by HHUS to be benign: 2 simple cysts, 1 hamartoma and 3 fibroadenomas on follow-up (Tables 1, 2).

Each breast mass was evaluated by both tomo and automated ultrasound according to the 5th edition of BIRADS lexicon.

\section{Tomosynthesis}

As regarding detection of the lesions, 1 (7.1\%) out of the 38 lesions was benign, and 3 lesions (7.9\%) were not detected by tomosynthesis, whereas 2 out of the 3 lesions (8.3\%) were malignant.

As regarding breast density, $6(15.8 \%)$ were ACR D, while 32 (84.2\%) were ACR C.

As regarding lesion shape, 10 (26.4\%) lesions were oval, 6 lesions were round $(15.8 \%$ ) [ 5 of them $=35.7 \%$ were benign and only 1 of them was malignant 4.2\%], and 19

Table 1 Age distribution among the studied patients

\begin{tabular}{llllllll}
\hline & Mean & Median & Minimum & Maximum & Standard deviation & Percentile 25 & Percentile 75 \\
\hline Age & 45 & 45 & 28 & 65 & 9 & 39 & 50 \\
\hline
\end{tabular}


Table 2 The histological diagnosis in the patients included in our study

\begin{tabular}{|c|c|c|c|}
\hline & & Count & $\%$ \\
\hline \multirow[t]{2}{*}{ Pathology } & Benign & 14 & 36.8 \\
\hline & Malignant & 24 & 63.2 \\
\hline \multirow[t]{2}{*}{ LN pathology } & Positive & 13 & 34.2 \\
\hline & Negative & 25 & 65.8 \\
\hline \multirow[t]{22}{*}{ Pathology details } & Adenocarcinoma & 1 & 2.6 \\
\hline & Bilateral adenosis with duct ectasia with insippisated secretions & 1 & 2.6 \\
\hline & Bilateral fibroadenoma & 1 & 2.6 \\
\hline & Complex cyst & 1 & 2.6 \\
\hline & Dilated ducts, lactating breast & 1 & 2.6 \\
\hline & Duct carcinoma in situ & 1 & 2.6 \\
\hline & Ductal carcinoma in situ with focal stromal invasion & 1 & 2.6 \\
\hline & Fibroadenoma & 2 & 5.3 \\
\hline & Fibroadenoma with complicated cyst & 1 & 2.6 \\
\hline & Fibrocystic disease & 2 & 5.3 \\
\hline & Hamartoma and galactocele & 1 & 2.6 \\
\hline & Intraductal papillary carcinoma with skin invasive & 1 & 2.6 \\
\hline & Invasive duct carcinoma & 11 & 28.9 \\
\hline & Invasive duct carcinoma mainly with vascular emboli & 1 & 2.6 \\
\hline & Invasive lobular carcinoma & 2 & 5.3 \\
\hline & Invasive mammary carcinoma & 2 & 5.3 \\
\hline & Mastitis & 1 & 2.6 \\
\hline & Metastatic carcinoma with focal glandular differentiation & 1 & 2.6 \\
\hline & Multiple cysts with macrocalcification & 1 & 2.6 \\
\hline & Periductal granulomatous mastitis & 1 & 2.6 \\
\hline & Positive lymph node & 1 & 2.6 \\
\hline & Simple cyst & 3 & 7.9 \\
\hline
\end{tabular}

(50.1\%) were irregular; all were pathologically proven malignant. The final correlation between the shape of the lesions at tomosynthesis and the final pathology was significant $(P$ value $<0.001)$.

As regarding the margin of the lesion, 14 (36.8\%) lesions were circumscribed [12 (85.7\%) were benign and 2 (8.3\%) were malignant], 1 (2.6\%) lesion had obscured benign margin, 1 (2.6\%) lesion was microlobulated, 2 (5.3\%) lesions had indistinct margins and they were all malignant, and 17 (44.7\%) lesions had speculated margins and were all malignant. The final correlation between the margin of the lesions at tomosynthesis and the final pathology was significant $(P$ value $<0.001)$.

As regarding the density of the lesion at tomosynthesis, no lesions had fat density, 6 lesions (15.8\%) had low density and were benign (100\%), 5 (13.2\%) lesions were of equal density [2 (14.3\%) were benign, 3 (12.5\%) were malignant], and 27 (71.1\%) lesions were of high density [6 (42.9\%) lesions were benign, 21 (87.5\%) lesions were malignant]. The final correlation between the margin of the lesions at tomosynthesis and the final pathology was significant ( $P$ value 0.002$)$.
As regarding asymmetry, there was no asymmetry in 30 cases $[14(100 \%)$ of them were benign and $16(69.6 \%)$ of them were malignant], $6(16.2 \%)$ cases showed global asymmetry and all of them were malignant (100\%), and $1(2.7 \%)$ case showed focal asymmetry and was malignant. The final correlation between asymmetry in tomosynthesis and the final pathology was insignificant ( $P$ value 0.072$)$.

As regarding calcification, no calcifications were detected in 23 lesions (60.5\%) [12 lesions (85.7\%) of them were benign and 11 were malignant (45.8\%)], 3 (7.9\%) lesions showed benign calcification at tomosynthesis (2 (14.3\%) lesions were benign and $1(4.2 \%)$ lesion was malignant], and 12 lesions (31.6\%) had suspicious amorphous calcifications and all were malignant (100\%). The final correlation between the morphology of calcifications in tomosynthesis and the final pathology was significant $(P$ value $<0.001)$.

As regarding the distribution of calcification, no calcifications were detected in 23 lesions (60.5\%) [12 lesions $(85.7 \%)$ of them were benign and 11 were malignant (45.8\%)], 5 (10.8\%) lesions showed diffuse calcification 
[3 (14.3\%) of them were benign and $2(8.7 \%)$ were malignant], 2 (2.7\%) lesion showed regional calcifications and was malignant, and 8 (21.6\%) lesions showed grouped calcifications (all were malignant 100\%). The final correlation between the distribution of calcifications in tomosynthesis and the final pathology was insignificant $(P$ value 0.065).

As regarding skin thickening and retraction at tomosynthesis, there was skin thickening in 10 lesions (26.3\%), while no skin thickening was noted in 28 lesions (73.7\%) [14 (100\%) of them were benign and $14(58.3 \%)$ were malignant]. There was a significant correlation between skin thickening and malignancy $P$ value $<0.001$.

As regarding nipple retraction, there were 6 (16.2\%) lesions with nipple retraction and 31 lesions with no nipple retraction (83.8\%). There was a significant correlation between significant nipple retraction and malignancy $(P$ value 0.037 ).

As regarding axillary lymphadenopathy, there was axillary lymphadenopathy in 11 cases $(29.7 \%)$ and they were pathologically proven malignant. There was no significant correlation between axillary lymphadenopathy and malignant pathology ( $P$ value 0.109 ).

As regarding architectural distortion, it was present in 8 lesions representing $21.1 \%$ and they were pathologically proven malignant. There was a significant correlation between architectural distortion and malignant pathology ( $P$ value 0.015$)$.

As regarding the number of lesions by tomosynthesis, no masses were detected in 3 cases (7.9\%), single lesion was detected in $21(55.3 \%)$ cases, and multiple lesions were detected in $14(36.8 \%)$ cases.

As regarding the BIRADS evaluation by tomosynthesis, 4 (10.5\%) lesions were considered BIRADS II, 1 (2.6\%) lesion was BIRADS 0 for further evaluation, 12 (31.6\%) lesions were reported BIRADS III, 7 (18.4\%) lesions were considered BIRADS 4, and 14 (36.8\%) lesions were considered BIRADS V by correlation with pathology (Fig. 1, Tables 3, 4, 5).

$$
\text { True positive }=14(100 \%) \quad \text { False positive }=3(12.5 \%)
$$$$
\text { False negative }=0(0.0 \%) \quad \text { True negative }=21(87.5 \%)
$$

\section{ABUS}

As regarding the shape of the lesion by ABUS, 13 (34.2\%) lesions were oval $[11(78.6 \%)$ lesions were benign and 2 $(8.3 \%)$ lesions were malignant], $3(7.9 \%)$ lesions were round and all were benign, and $22(57.9 \%)$ lesions were irregular and all were malignant (78.8\%). There was a significant correlation between the shape of the lesion by ABUS and pathology $(P$ value $<0.001)$.

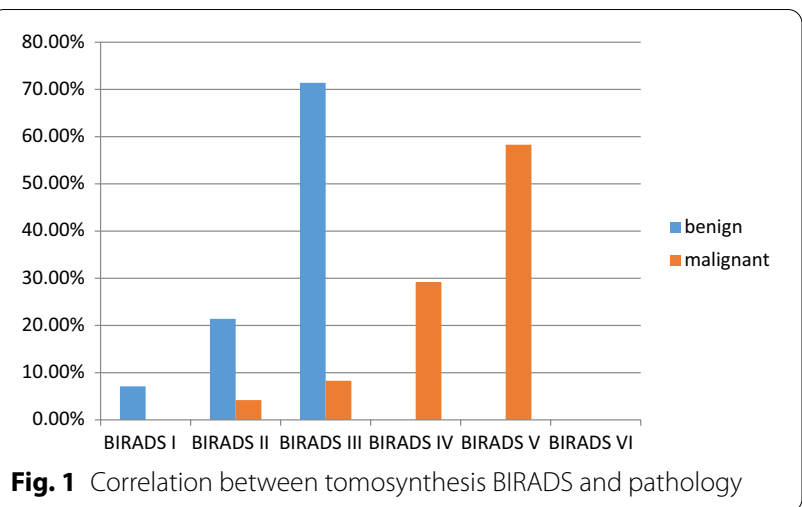

Table 3 Characterizations of lesions in tomosynthesis

\begin{tabular}{|c|c|c|c|}
\hline & & Count & $\%$ \\
\hline \multirow[t]{4}{*}{ ACR (TOMO) } & ACR A & 0 & 0.0 \\
\hline & $A C R B$ & 0 & 0.0 \\
\hline & ACR C & 32 & 84.2 \\
\hline & ACR D & 6 & 15.8 \\
\hline \multirow[t]{4}{*}{ Shape (TOMO) } & Oval & 10 & 26.3 \\
\hline & Round & 6 & 15.8 \\
\hline & Irregular & 19 & 50.0 \\
\hline & No mass & 3 & 7.9 \\
\hline \multirow[t]{6}{*}{ Margin (TOMO) } & Circumscribed & 14 & 36.8 \\
\hline & Obscured & 1 & 2.6 \\
\hline & Microlobulated & 1 & 2.6 \\
\hline & Indistinct & 2 & 5.3 \\
\hline & Speculated & 17 & 44.7 \\
\hline & No mass & 3 & 7.9 \\
\hline \multirow[t]{4}{*}{ Density (TOMO) } & Fat & 0 & 0.0 \\
\hline & Low & 6 & 15.8 \\
\hline & Equal & 5 & 13.2 \\
\hline & High & 27 & 71.1 \\
\hline \multirow[t]{4}{*}{ Asymmetry (TOMO) } & No asymmetry & 30 & 81.1 \\
\hline & Global & 6 & 16.2 \\
\hline & Focal & 1 & 2.7 \\
\hline & Developing & 0 & 0.0 \\
\hline \multirow[t]{3}{*}{ Calcification (TOMO) } & No calcification & 23 & 60.5 \\
\hline & Bengin & 3 & 7.9 \\
\hline & Suspicious & 12 & 31.6 \\
\hline \multirow[t]{6}{*}{ Distribution of calcification (TOMO) } & No calcification & 24 & 64.9 \\
\hline & Diffuse & 4 & 10.8 \\
\hline & Regional & 1 & 2.7 \\
\hline & Grouped & 8 & 21.6 \\
\hline & Linear & 0 & 0.0 \\
\hline & Segmental & 0 & 0.0 \\
\hline \multirow[t]{2}{*}{ Nipple retraction (TOMO) } & Yes & 6 & 16.2 \\
\hline & No & 31 & 83.8 \\
\hline
\end{tabular}


Table 4 Correlation between pathology results and tomosynthesis final results

\begin{tabular}{|c|c|c|c|c|c|c|c|c|}
\hline & & \multicolumn{6}{|c|}{ Pathology } & \multirow[t]{3}{*}{$P$ value } \\
\hline & & \multicolumn{2}{|l|}{ Benign } & \multicolumn{2}{|c|}{ Malignant } & \multicolumn{2}{|l|}{ Total } & \\
\hline & & Count & $\%$ & Count & $\%$ & Count & $\%$ & \\
\hline \multirow[t]{2}{*}{ TOMO } & Benign & 14 & 100.0 & 3 & 12.5 & 17 & 44.7 & $<0.001$ \\
\hline & Malignant & 0 & 0.0 & 21 & 87.5 & 21 & 55.3 & \\
\hline
\end{tabular}

Table $\mathbf{5}$ Tomosynthesis accuracy measures

\begin{tabular}{lc}
\hline Tomosynthesis statistics & Value (\%) \\
\hline Sensitivity & 87.5 \\
Specificity & 100.0 \\
Positive predictive value & 100.0 \\
Negative predictive value & 82.4 \\
Accuracy & 92.1 \\
\hline
\end{tabular}

As regarding the margin of the lesion by ABUS, 14 (36.8\%) lesions were circumscribed [13 (92.9\%) lesions were benign and 1 (4.2\%) lesion was malignant], 3 (7.9\%) lesions had indistinct margins and all were malignant, 2 (5.3\%) lesions had angular borders [1 lesion (7.1\%) was benign and 1 lesion (4.2\%) was malignant], 19 (50\%) lesions had speculated margins and (100\%) all of them were malignant). There was a significant correlation between the margin of the lesion by ABUS and pathology $(P$ value $<0.001)$.

As regarding orientation of the lesion, 15 (40.5\%) lesions were parallel [14 (100\%) lesions were benign and 1 (4.3\%) lesion was malignant] and 22 (59.5\%) lesions were non-parallel and all were malignant. There was a significant correlation between orientation of the lesion by ABUS and pathology $(P$ value $<0.001)$.

As regarding echo pattern of the lesion, 1 (2.7\%) lesion was anechoic and it was benign, 29 (78.4\%) lesions were hypoechoic $[10(71.4 \%)$ lesions were benign and 19 $(82.6 \%)$ were malignant], $3(8.1 \%)$ lesions were isoechoic [ 1 (7.1\%) was benign and $2(8.7 \%)$ were malignant], $3(8.1 \%)$ lesions were heterogeneous [ 2 were benign (14.3\%) and 1 (4.3\%) was malignant], and 1 (2.7\%) lesion was benign complex solid and cyst. There was an insignificant correlation between lesion echo pattern by ABUS and pathology ( $P$ value 0.481$)$.

As regarding posterior features of the lesions, no posterior features in 23 lesions (62.2\%) [12 (85.7\%) lesions were benign and 11 (47.8\%) were malignant], 6 (16.2\%) lesions showed posterior enhancement [2 (14.3\%) were benign and $4(17.4 \%)$ were malignant], and 7 (18.9\%) lesions showed posterior shadowing and all were malignant. One (2.7\%) lesion showed combined pattern of posterior shadowing and enhancement, and it was malignant (100\%). There was an insignificant correlation between the posterior features of the lesion by ABUS and pathology ( $P$ value 0.074$)$.

As regarding calcifications of the lesions, there were no calcifications in 37 (97.4\%) lesions, and there was calcification in 1 lesion (2.6\%). There was an insignificant correlation between calcification in mass and malignancy $(P$ value 0.439 ).

As regarding axillary lymphadenopathy detection by ABUS, axillary lymphadenopathy was detected by ABUS in 8 (21.1\%) [2 (14.3\%) were benign and 6 (25\%) were malignant], while no axillary lymphadenopathy was seen in $30(78.9 \%)$ cases [12 $(85.7 \%)$ were benign, while 18 (75\%) were malignant]. There was no significant correlation between axillary lymphadenopathy by ABUS and pathology ( $P$ value 0.435$)$.

As regarding margin of the lesions in coronal view that is unique view for ABUS examination, 15 (39.5\%) lesions showed complete hyperechoic rim [12 (85.7\%) were benign) and 3 (12.5\%) were malignant], 5 (13.2\%) lesions showed retraction phenomenon (all were malignant $100 \%$ ), and 18 (47.4\% lesions showed incomplete (discontinuous) hyperechoic rim [2 (14.3\%) were benign, while $16(66.7 \%)$ were malignant]. Specificity of retraction phenomena for malignant lesions was $100 \%$, while sensitivity was $75 \%$. The sensitivity of hyperechoic rim for benign lesions was $52.8 \%$, while the specificity was $90.5 \%$.

As regarding numbers of the lesion by ABUS, no masses were detected in $3(7.9 \%)$ cases, single lesion was detected in 17 (44.7\%) cases, and multiple lesions were detected in 18 (47.4\%) cases.

Eleven lesions (28.9\%) showed skin thickening by ABUS and all were malignant (100\%), while no skin thickening was noted in 27 (71.1\%) lesions [14 (100\%) were benign, while $13(54.2 \%)$ were malignant]. The correlation between skin thickening and malignant pathology was significant ( $P$ value 0.003 ) (Figs. $2,3,4,5$, Tables 6,7 , $8,9,10,11,12)$.

Eight lesions (21.6\%) showed nipple retraction by ABUS, and all were malignant (100\%). While no nipple retraction was noted in $29(78.4 \%)$ lesions [14 (100\%) were benign, while $15(65.2 \%)$ were malignant], the 


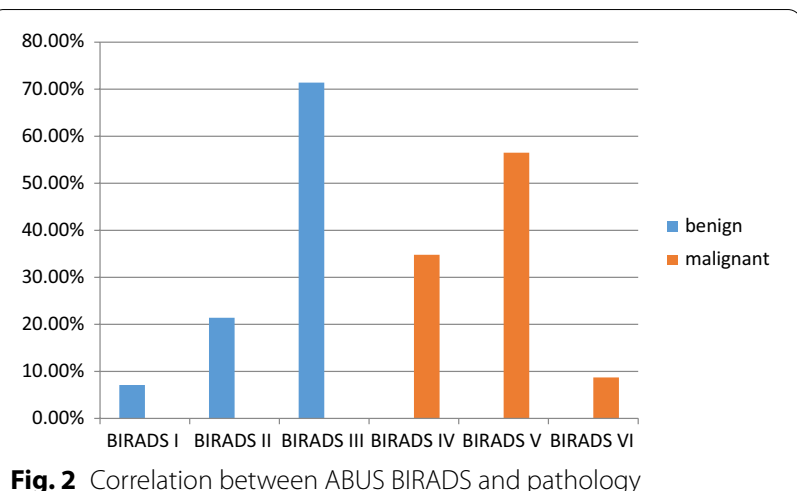

Fig. 2 Correlation between ABUS BIRADS and pathology

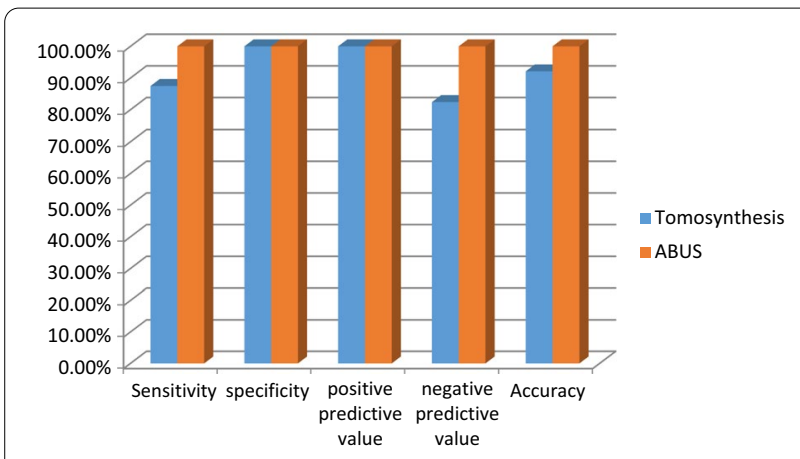

Fig. 3 Comparison between accuracy measures of tomosynthesis and $A B \cup S$

correlation between nipple retraction and malignant pathology was significant ( $P$ value 0.013 ).

False positive $=0(0 \%) \quad$ True positive $=14(100 \%)$

True negative $=23(100 \%) \quad$ False negative $=0(0 \%)$

This table shows a positive significant correlation between the size of the lesions at tomosynthesis and ABUS (0.4). The intraclass correlation coefficient as regarding the size of the lesions (longest dimension) between ABUS and tomosynthesis is 0.325 . There is no significant correlation between lesion distance from nipple at tomosynthesis and ABUS $(P$ value $=0.330)$. There is no significant correlation between lesion distance from skin at tomosynthesis and ABUS ( $P$ value $=0.776)$.

\section{Discussion}

The role of breast imaging radiologist is very important as cancer breast is the most common malignancy in both developing and developed countries, so mammography remains the gold standard imaging in screening and early detection [2].

As a result of the 3D imaging capabilities of tomosynthesis, it plays an important role in screening and diagnosis of breast lesions, alleviating the limitation of mammography in masking cancers by dense breast tissue [3]. Tomosynthesis provides better imaging quality and accuracy in detection and classification of the lesions [4].

Ultrasonography is used in conjugation with mammography in detection and assessment of breast lesions as it is an inexpensive, noninvasive and non-ionizing imaging modality. However, it is operator dependent and image evaluation should be done during the procedure [5].

Nowadays, ABUS is used for breast evaluation, as it is an operator-independent US imaging with a high-resolution transducer [5].

The study of Poplack et al. [6], which included 98 women with abnormal mammography, had compared mammography with tomosynthesis in image quality and recall rates of screening. The results reported that tomosynthesis has superior or comparable image quality to that of mammography and reduces the recall rate when used with digital screening mammography [6].

Andersson et al. [7]'s study, in Canada on 36 patients, compared the breast cancer visibility in one-view tomosynthesis to cancer visibility in one- or two-view digital mammography (DM). The results indicated that the cancer visibility on breast tomosynthesis (BT) is superior to DM, which suggests that BT may have a higher sensitivity for breast cancer detection [7].

A comparison had been made in a study done by Gennaro et al. [8] in Italy, between the full-field digital mammography (FFDM) and the clinical performance of digital breast tomosynthesis (DBT), which showed that the clinical performance of tomosynthesis in one view at the same total dose as standard screen-film mammography is not inferior to digital mammography in two views [8].

Hakim et al. [9] examined 25 females and compared DBT to the additional mammographic views in different lesions characterization, asymmetries and architecture distortion and concluded that DBT could be an alternative to additional mammographic views in most patients.

Spangler et al. [10] proved in a study done on 100 patients that the FFDM is still more sensitive than digital tomosynthesis in detection and characterization of calcifications.

However, a study made by Zhang et al. [11] on a large sample (182) reported that tomosynthesis had more diagnostic accuracy for non-calcified lesions in comparison with additional mammographic views.

Teertstra et al. [12] study was done in the Netherlands on 519 cases and proved that tomosynthesis can be used as an additional technique to mammography in patients referred with an abnormal screening mammogram or with clinical symptoms.

A study was done by Kim et al. [13] on 119 patients, which proved that digital breast tomosynthesis could 

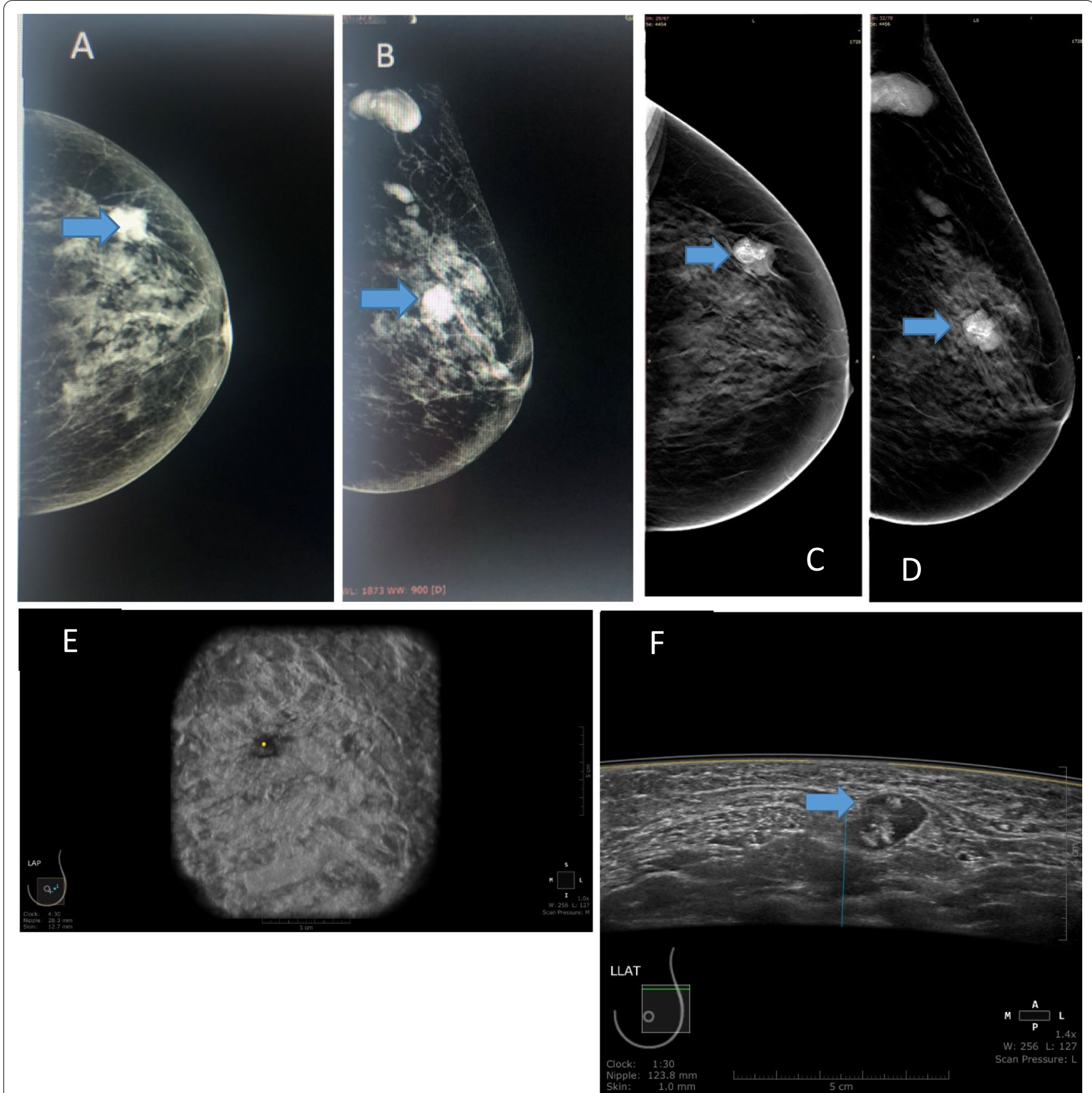

Fig. 4 A and B: Digital Mx CC and MLO views of the right breast show an oval-shaped circumscribed high-density UOQ mass with no associated skin thickening. $\mathbf{C}$ and $\mathbf{D}$ : Tomosynthesis CC and MLO views of the right breast confirmed the mammographic findings, however, with better delineation of the margins (BIRADS IV). E: Automated ultrasound right AP coronal image shows small focal defect at distance $28.3 \mathrm{~mm}$ from the nipple. F: Automated ultrasound image shows a right rather well-defined mass, non-parallel orientation and heterogeneous echo pattern (BIRADS V). Core biopsy was done and revealed adenocarcinoma. Automated ultrasound was superior to tomosynthesis in characterization of the lesion, while tomosynthesis was superior in detection of right axillary lymphadenopathy

detect similar lesion characterization performance compared to that of US for the lesions depicted on digital mammography (DM).

The retrospective study of Krammer et al. [14] proved that digital breast tomosynthesis (DBT) could improve the preoperative breast cancer staging in dense breast patients compared to conventional mammography alone. However, limitations have to be expected in the case of invasive lobular carcinoma [14]. 

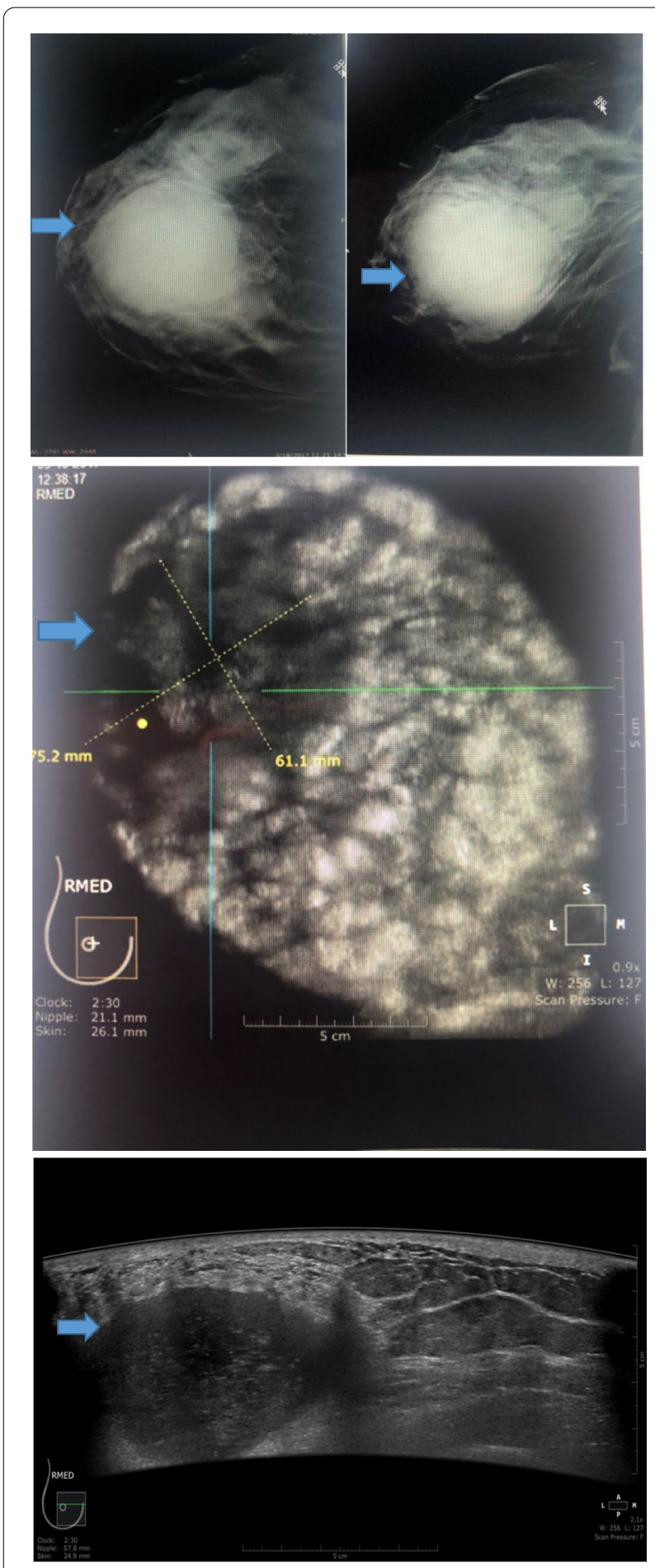

Kelly et al. [15] compared the mammographic diagnostic performance to that of the automated breast ultrasound (ABUS) in dense breast and resulted in significant cancer detection improvement by ABUS compared with mammography alone.
Fig. 5 A 42-year-old female patient presenting with tender right breast. Tomosynthesis CC and MLO views of the right breast confirmed the previously described mammographic findings (not shown), however, with less fibroglandular tissue overlap and thus better delineation of the margins (BIRADS III). Automated ultrasound right medial coronal image shows a large defect at 2:30 o'clock. Automated ultrasound right medial axial image shows a right oval-shaped mass with indistinct margin, parallel orientation and hypoechoic echo genic cyst (BIRADS III). The lesion was proved to be inflammatory mastitis with acute abscess formation by aspiration of the abscess content. Automated ultrasound was superior to tomosynthesis in predicting the exact pathology

Another study retrospectively depicted that ABUS had higher performance in detecting malignant lesions with their surrounding changes than benign lesions [16].

The study of Lin et al. [17], which was performed in China on 81 cases, compared the clinical utility of ABUS against HHUS in breast lesion detection and proved that ABUS had high diagnostic accuracy, operator independence and better lesion size.

A similar study was conducted on 175 patients, and it evaluated the differences in the diagnostic values of the conventional handheld B-mode ultrasound (HHUS) and automated breast ultrasound (ABUS) for benign and malignant breast masses and compared it with the final pathologic findings. It showed that overall ABUS and HHUS do not differ in diagnostic accuracy for the differentiation of malignant or benign breast masses [5].

Giuliano et al. [18] study was performed in 3418 asymptomatic women with mammographically detected dense breasts and proved that ABUS could be used in conjunction with mammography in the dense breast screening population study.

Giger et al. [19] compared the performance of breast cancer detection using full-field digital mammography (FFDM) alone and using FFDM with ABUS. The study included 185 cases, 133 non-cancers and 52 biopsyproven cancers. They concluded that combining mammography with ABUS compared with mammography alone had significantly improved readers' detection of breast cancers in women with dense breast tissue without substantially affecting specificity [19].

The current research was a prospective study conducted on 38 patients presenting with dense breast masses either discovered clinically or discovered by mammographic examination as a part of the early detection program. They have been evaluated by means of digital breast tomosynthesis and automated breast ultrasound, 38 masses were detected in the 38 patients, and pathological confirmation was done in most masses $(n=31 / 38)$ and ultrasonography confirmation in the rest 
Table 6 Characterizations of the lesions (ABUS)

\begin{tabular}{|c|c|c|c|}
\hline & & Count & $\%$ \\
\hline \multirow[t]{3}{*}{ Breast composition (automated) } & Homogenous fat & 0 & 0.0 \\
\hline & Homogenous fibroglandular & 28 & 75.7 \\
\hline & Heterogenous & 9 & 24.3 \\
\hline \multirow[t]{3}{*}{ Shape (automated) } & Oval & 13 & 34.2 \\
\hline & Round & 3 & 7.9 \\
\hline & Irregular & 22 & 57.9 \\
\hline \multirow[t]{5}{*}{ Margin (automated) } & Circumscribed & 14 & 36.8 \\
\hline & Indistinct & 3 & 7.9 \\
\hline & Angular & 2 & 5.3 \\
\hline & Microlobulated & 0 & 0.0 \\
\hline & Speculated & 19 & 50.0 \\
\hline \multirow[t]{2}{*}{ Orientation (automated) } & Parallel & 15 & 40.5 \\
\hline & Not parallel & 22 & 59.5 \\
\hline \multirow[t]{6}{*}{ Echo pattern (automated) } & Anechoic & 1 & 2.7 \\
\hline & Hyper-echoic & 0 & 0.0 \\
\hline & Complex solid and cystic & 1 & 2.7 \\
\hline & Hypoechoic & 29 & 78.4 \\
\hline & Isoechoic & 3 & 8.1 \\
\hline & Heterogenous & 3 & 8.1 \\
\hline \multirow{4}{*}{ Posterior feature (automated) } & No features & 23 & 62.2 \\
\hline & Enhancement & 6 & 16.2 \\
\hline & Shadowing & 7 & 18.9 \\
\hline & Combined pattern & 1 & 2.7 \\
\hline \multirow[t]{4}{*}{ Calcification (automated) } & No calcification & 37 & 97.4 \\
\hline & In mass & 1 & 2.6 \\
\hline & Outside mass & 0 & 0.0 \\
\hline & Intra-ductal & 0 & 0.0 \\
\hline \multirow[t]{3}{*}{ Mass N (automated) } & Single & 17 & 44.7 \\
\hline & Multiple & 18 & 47.4 \\
\hline & No & 3 & 7.9 \\
\hline \multirow[t]{7}{*}{ Location (automated) } & UOQ & 18 & 47.4 \\
\hline & UIQ & 2 & 5.3 \\
\hline & LOQ & 2 & 5.3 \\
\hline & LIQ & 1 & 2.6 \\
\hline & Retroareolar & 7 & 18.4 \\
\hline & Scattered & 7 & 18.4 \\
\hline & Axilla & 1 & 2.6 \\
\hline \multirow[t]{2}{*}{ Nipple retraction (automated) } & Yes & 8 & 21.6 \\
\hline & No & 29 & 78.4 \\
\hline \multirow[t]{2}{*}{ Skin thickening and retraction (automated) } & Yes & 11 & 28.9 \\
\hline & No & 27 & 71.1 \\
\hline \multirow[t]{2}{*}{ Axillary lymphadenopathy (automated) } & Yes & 8 & 21.1 \\
\hline & No & 30 & 78.9 \\
\hline \multirow[t]{2}{*}{ Laterality (automated) } & Unilateral & 22 & 57.9 \\
\hline & Bilateral & 16 & 42.1 \\
\hline \multirow[t]{3}{*}{ AUTO character (automated) } & Solid & 17 & 45.9 \\
\hline & Cystic & 9 & 24.3 \\
\hline & Solid with cystic component & 11 & 29.7 \\
\hline
\end{tabular}


Table 6 (continued)

\begin{tabular}{|c|c|c|c|}
\hline & & Count & $\%$ \\
\hline \multirow[t]{3}{*}{ Coronal view (automated) } & Retraction phenomena & 5 & 13.2 \\
\hline & Complete hyperechoic rim & 15 & 39.5 \\
\hline & Incomplete hyperechoic rim & 18 & 47.4 \\
\hline \multirow[t]{2}{*}{ Extension (automated) } & Yes & 9 & 23.7 \\
\hline & No & 29 & 76.3 \\
\hline \multirow[t]{6}{*}{ BIRADS (automated) } & BIRADS I & 1 & 2.7 \\
\hline & BIRADS II & 3 & 8.1 \\
\hline & BIRADS III & 10 & 27.0 \\
\hline & BIRADS IV & 8 & 21.6 \\
\hline & BIRADS V & 13 & 35.1 \\
\hline & BIRADS VI & 2 & 5.4 \\
\hline
\end{tabular}

Table 7 Correlation between ABUS BIRADS and pathology

\begin{tabular}{|c|c|c|c|c|c|c|c|c|}
\hline & & \multicolumn{6}{|c|}{ Pathology } & \multirow[t]{3}{*}{$P$ value } \\
\hline & & \multicolumn{2}{|c|}{ Benign } & \multicolumn{2}{|c|}{ Malignant } & \multicolumn{2}{|l|}{ Total } & \\
\hline & & Count & $\%$ & Count & $\%$ & Count & $\%$ & \\
\hline \multirow[t]{2}{*}{ Automated } & Benign & 14 & 100.0 & 0 & 0.0 & 14 & 37.8 & \multirow[t]{2}{*}{$<0.001$} \\
\hline & Malignant & 0 & 0.0 & 23 & 100.0 & 23 & 62.2 & \\
\hline
\end{tabular}

Table 8 ABUS accuracy measures

\begin{tabular}{ll}
\hline ABUS statistics & Value (\%) \\
\hline Sensitivity & 100.0 \\
Specificity & 100.0 \\
Positive predictive value & 100.0 \\
Negative predictive value & 100.0 \\
Accuracy & 100.0 \\
\hline
\end{tabular}

Table 9 Comparison between accuracy measures of ABUS and tomosynthesis

\begin{tabular}{lcc}
\hline Statistic & Tomosynthesis (\%) & ABUS \\
\hline Sensitivity & 87.5 & 100.0 \\
Specificity & 100.0 & 100.0 \\
Positive predictive value & 100.0 & 100.0 \\
Negative predictive value & 82.4 & 100.0 \\
Accuracy & 92.1 & 100.0 \\
\hline
\end{tabular}

( $n=7$ /38): simple cysts, fat containing lesion (hamartoma) and fibroadenomas.

Image data analysis from both automated ultrasound and tomosynthesis was performed by two radiologists independently, and the findings were reported as per the morphology descriptors used in the BIRADS lexicon. The data were interpreted as regards the location, extension of the lesions, mass number, shape, margin, skin retraction and thickening, and the presence of calcification, and a final score was given according to the BIRADS assessment categories. The specificity and sensitivity of each modality in characterization and detection of breast lesions were detected.

Our study included 38 females presenting with different types of breast lesions.

Thirty-eight masses were detected, among which 14 were benign $(n=14 / 38)$ and 24 were malignant $(n=24 / 38)$.

Histopathology of the masses was reached using different techniques such as core biopsy $(n=24)$, FNAC $(n=6)$ and excisional biopsy $(n=2)$. The rest of the masses ( $n=6 / 38$ ) were confirmed by their characteristic sonographic appearances: anechoic simple cysts and mixed echogenicity hamartoma.

Different pathologies were identified, some of which were benign such as fibroadenoma, simple cysts, hamartoma, inflammatory mastitis and focal fibroadenosis. The latter was falsely diagnosed by tomosynthesis as a mass due to its circumscribed margin.

Others were malignant, such as invasive duct carcinoma (commonest type of malignancy in our study $28.9 \%$ ), invasive lobular carcinoma, invasive mammary 
Table 10 Agreement between tomosynthesis and ABUS BIRADS

\begin{tabular}{|c|c|c|c|c|c|c|}
\hline & & \multicolumn{2}{|c|}{ BIRADS Automated } & \multirow[t]{2}{*}{ Total } & \multirow[t]{2}{*}{ Kappa } & \multirow[t]{2}{*}{$P$ value } \\
\hline & & Benign & Malignant & & & \\
\hline \multicolumn{7}{|c|}{ BIRADS TOMO } \\
\hline \multirow[t]{2}{*}{ Benign } & Count & 14 & 3 & 17 & 0.835 & $<0.001$ \\
\hline & $\%$ within BIRADSAutomated2 & $100.0 \%$ & $13.0 \%$ & $45.9 \%$ & & \\
\hline \multirow[t]{2}{*}{ Malignant } & Count & 0 & 20 & 20 & & \\
\hline & $\%$ within BIRADSAutomated2 & $0.0 \%$ & $87.0 \%$ & $54.1 \%$ & & \\
\hline \multicolumn{7}{|l|}{ Total } \\
\hline & Count & 14 & 23 & 37 & & \\
\hline & \% Within BIRADSAutomated2 & $100.0 \%$ & $100.0 \%$ & $100.0 \%$ & & \\
\hline
\end{tabular}

Table 11 Correlation between lesion size and pathology at tomosynthesis

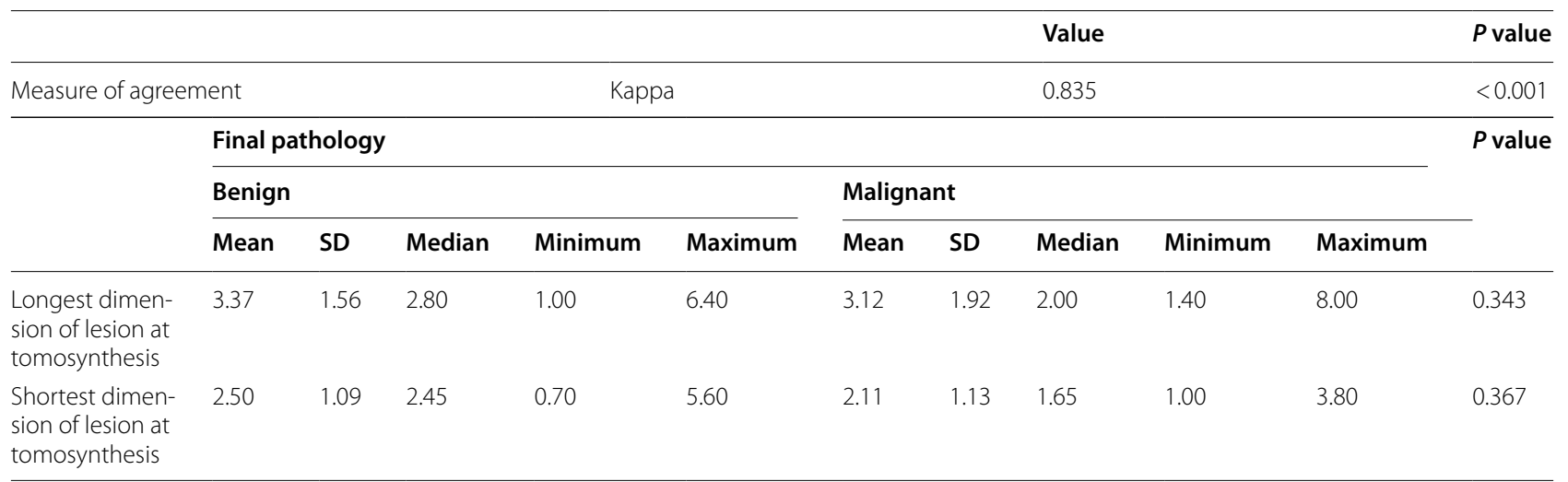

Table 12 Correlation between lesion size and pathology at ABUS

\begin{tabular}{|c|c|c|c|c|c|c|c|c|c|c|c|}
\hline & \multicolumn{10}{|c|}{ Final pathology } & \multirow[t]{3}{*}{$P$ value } \\
\hline & \multicolumn{5}{|c|}{ Benign } & \multicolumn{5}{|c|}{ Malignant } & \\
\hline & Mean & SD & Median & Minimum & Maximum & Mean & SD & Median & Minimum & Maximum & \\
\hline $\begin{array}{l}\text { Longest } \\
\text { dimension of } \\
\text { lesion at ABUS }\end{array}$ & 2.56 & 1.14 & 2.40 & 0.70 & 6.00 & 2.70 & 1.17 & 2.70 & 0.60 & 5.00 & 0.580 \\
\hline $\begin{array}{l}\text { Shortest } \\
\text { dimension of } \\
\text { lesion at ABUS }\end{array}$ & 1.33 & 0.56 & 1.30 & 0.40 & 3.00 & 1.61 & 0.60 & 1.50 & 0.30 & 3.00 & 0.055 \\
\hline
\end{tabular}

carcinoma and duct carcinoma in situ. The latter was the most difficult to detect in this study due to the subtle changes of ductal dilatation.

Among our cases, two were metastatic breast masses. One of them was female patient who gave a history of cancer colon; the mass appeared irregular with speculated margins, so malignant nature was suspected, then core biopsy was done, and histopathology revealed metastatic adenocarcinoma. On the contrary, the other metastatic breast mass was circumscribed with no available data regarding any history of malignancy, and histopathology revealed metastatic melanoma.

Six masses were diagnosed by their characteristic sonographic findings; 2 of them were simple cysts with the characteristic anechoic nature and posterior enhancement, and 1 was hamartoma with their special imaging features (fat containing mass which resembles breast within the breast), and 3 were fibroadenomas with characteristic hypoechoic oval lesion. 
According to the newest BIRADS lexicon criteria in the description of malignant masses, 22 masses were irregular on both modalities and were proved to be malignant, yet tomosynthesis underestimated one case since it showed rounded mass (normally to a benign descriptor). On the other hand, fine speculation was detected on automated ultrasound images and proved by core biopsy to be malignant in nature.

We found out that the sensitivity of ABUS was 87\% regarding the malignant lesion shape characterization in comparison with tomosynthesis which showed $69.6 \%$ sensitivity.

Regarding the margins, $92.9 \%$ of the circumscribed masses were benign and $100 \%$ of the speculated masses were malignant in our study. Despite that three masses were circumscribed on both modalities, their histopathology results proved them malignant. This confirmed that a single criterion was not enough for radiologists to reach a correct final diagnosis. Tomosynthesis showed 87.5\% sensitivity, while ABUS showed 100\% sensitivity in the current study.

Regarding the number of masses, tomosynthesis detected multiple lesions in about 14 out of 18 cases, while automated ultrasound was able to detect the whole 18 cases.

Although most of the masses were seen on both modalities, tomosynthesis missed one case, yet it was visible by automated ultrasound as a very small irregular mass.

ABUS showed $23.7 \%$ in the category of detecting mass extension, while tomosynthesis showed $31.6 \%$.

For the skin thickening and retraction, tomosynthesis was sensitive in $26.3 \%$, while automated ultrasound was sensitive in $28.9 \%$.

As regards the final BIRADS score given for both modalities, tomosynthesis showed an accuracy of $92.1 \%$ in characterization of malignant masses with an accuracy of $100 \%$ in benign masses; on the other hand, automated ultrasound showed 100\% accuracy in characterization of malignant masses with $100 \%$ accuracy in benign masses.

In summary, we detected in the current study that the sensitivity of tomosynthesis in characterization and detection of breast lesions was $87.5 \%$; both the specificity and PPV were $100 \%$, while the negative predictive value was $82.4 \%$. On the other hand, ABU showed $100 \%$ in all the sensitivity, the specificity, PPV and NPV.

Our study succeeded to prove that ABUS is a reliable screening and diagnostic imaging tool. It could detect different breast lesions and abnormalities whether benign or malignant, showed improvements in cancer detection rates, decreased the need for further assessments, reduced the false positive rates, and showed superior performance compared to digital mammography, in lesion detection, characterization, tumor margins and extent, multifocality and multi-centricity of the malignant lesions, and image quality, especially in dense breast lesions.

\section{Conclusion}

ABUS in addition to tomosynthesis can make a revolution in breast screening and diagnostic performance, specially in women with dense breasts.

\section{Abbreviations}

ABUS: Automated breast ultrasound; DBT: Digital breast tomosynthesis; HHUS: Handheld ultrasound; BIRADS: Breast Imaging and Reporting Data System.

\section{Acknowledgements}

First and foremost, thanks are due to Allah, the most beneficial and most merciful. It is but for his mercy that we can put through in life.

\section{Authors' contributions}

EA, FS and LA analyzed and interpreted the patient data according to the mammographic examination and wrote the manuscript. All authors have read and approved the manuscript.

\section{Funding}

Not applicable.

\section{Availability of data and materials}

The datasets used and/or analyzed during the current study are available from the corresponding author on reasonable request.

\section{Declarations}

\section{Ethics approval and consent to participate}

The study is a prospective study that was reviewed by the ethics committee of radiology departments at National Cancer Institute and Kasr Al-Ainy Hospital and was approved by the review board that is related to our University.

Patients included in this research gave informed written consent to use their data in research work. Reference number is not applicable

\section{Consent for publication}

All the patients included in this research gave written informed consent to publish the data contained within this study.

\section{Competing interests}

The authors declare that they have no competing interests.

\section{Author details}

${ }^{1}$ Diagnostic and Intervention Radiology Department (Women's Imaging Unit), Kasr El-Ainy Hospital, Cairo University Hospitals, El-Manial, Cairo 11956, Egypt.

${ }^{2}$ Diagnostic and Intervention Radiology Department, Cancer of Institute, Cairo University Hospitals, Cairo 11956, Egypt.

Received: 4 April 2021 Accepted: 11 November 2021

Published online: 24 November 2021

\section{References}

1. Berg WA, Gutierrez L, NessAiver MS et al (2004) Diagnostic accuracy of mammography, clinical examination, US, and MR imaging in preoperative assessment of breast cancer. Radiology 233(3):830-849

2. Coughlin SS, Ekwueme DU (2009) Breast cancer as a global health concern. Cancer Epidemiol 33(5):315-318

3. Helvie M (2010) Digital mammography imaging: breast tomosynthesis and advanced applications. Radiol Clin N Am 48(5):917-929. https://doi. org/10.1016/j.rcl.2010.06.009 
4. Gennaro G, Hendrick R, Toledano A et al (2013) Combination of one-view digital breast tomosynthesis with one-view digital mammography versus standard two-view digital mammography: per lesion analysis. Eur Radiol 23(8):2087-2094

5. Chen L, Chen Y, Diao XH, Fang L, Pang Y, Cheng AQ, Li WP, Wang Y (2013) Comparative study of automated breast 3-D ultrasound and handheld B-mode ultrasound for differentiation of benign and malignant breast masses. Ultrasound Med Biol 39(10):1735-1742

6. Poplack SP, Tosteson TD, Kogel CA, Nagy HM (2007) Digital breast Tomosynthesis: initial experience in 98 women with abnormal digital screening mammography. Am J Roentgenol 189(3):616-623

7. Andersson I, Ikeda D, Zackrisson S et al (2008) Breast Tomosynthesis and digital mammography: a comparison of breast cancer visibility and BIRADS classification in a population of cancers with subtle mammographic findings. Eur Radiol 18:2817-2825

8. Gennaro G, Toledano A, Di Maggio C, Baldan E, Bezzon E, La Grassa M, Pescarini L, Polico I, Proietti A, Toffoli A, Muzzio PC (2010) Digital breast tomosynthesis versus digital mammography: a clinical performance study. Eur Radiol 20(7):1545-1553

9. Hakim CM, Chough DM, Ganott MA, Sumkin JH, Zuley ML, Gur D (2010) Digital breast tomosynthesis in the diagnostic environment: a subjective side-by-side review. Am J Roentgenol 195(2):W172-W176

10. Spangler M, Zuley M, Sumkin J et al (2011) Detection and classification of calcifications on digital breast tomosynthesis and 2D digital mammography: a comparison. Am J Roentgenol 196(2):320-324

11. Zhang Q, Hu B, Hu B et al (2013) Detection of breast lesions using an automated breast volume scanner system. J Int Med Res 40(1):300-330

12. Teertstra H, Loo C, Van Den Bosch M et al (2010) Breast Tomosynthesis in clinical practice: initial results. Eur Radiol 20(1):16-24
13. Kim SA, Chang JM, Cho N, Yi A, Moon WK (2015) Characterization of breast lesions: comparison of digital breast tomosynthesis and ultrasonography. Korean J Radiol 16(2):229-238

14. Krammer J, Stepniewski K, Kaiser CG, Brade J, Riffel P, Schoenberg SO, Wasser K (2017) Value of additional digital breast Tomosynthesis for preoperative staging of breast cancer in dense breasts. Anticancer Res 37(9):5255-5261

15. Kelly KM, Dean J, Comulada WS, Lee SJ (2010) Breast cancer detection using automated whole breast ultrasound and mammography in radiographically dense breasts. Eur Radiol 20(3):734-742

16. Chang JM, Moon WK, Cho N, Park JS, Kim SJ (2011) Radiologists' performance in the detection of benign and malignant masses with 3D automated breast ultrasound (ABUS). Eur J Radiol 78(1):99-103

17. Lin X, Wang J, Han F, Fu J, Li A (2012) Analysis of eighty-one cases with breast lesions using automated breast volume scanner and comparison with handheld ultrasound. Eur J Radiol 81(5):873-878

18. Giuliano V, Giuliano C (2013) Improved breast cancer detection in asymptomatic women using 3D-automated breast ultrasound in mammographically dense breasts. Clin Imaging 37(3):480-486

19. Giger ML, Inciardi MF, Edwards A, Papaioannou J, Drukker K, Jiang Y, Brem R, Brown JB (2016) Automated breast ultrasound in breast cancer screening of women with dense breasts: reader study of mammography-negative and mammography-positive cancers. Am J Roentgenol 206(6):1341-1350

\section{Publisher's Note}

Springer Nature remains neutral with regard to jurisdictional claims in published maps and institutional affiliations.

\section{Submit your manuscript to a SpringerOpen ${ }^{\circ}$ journal and benefit from:}

- Convenient online submission

- Rigorous peer review

- Open access: articles freely available online

- High visibility within the field

Retaining the copyright to your article

Submit your next manuscript at springeropen.com 